\title{
Is a yoga-based program with potential to decrease falls perceived to be acceptable to community-dwelling people older than 60?
}

\author{
Anne Tiedemannn a,b, Sandra O'Rourke ${ }^{a}$ and Catherine Sherrington ${ }^{a}$
}

a Sydney School of Public Health, University of Sydney, NSW, Australia

b Corresponding author: anne.tiedemann@sydney.edu.au

\section{Article history}

Publication date: June 2018

Citation: Tiedemann A, O'Rourke S,

Sherrington $C$. Is a yoga-based program with potential to decrease falls perceived to be acceptable to community-dwelling people older than 60? Public Health Res Pract. 2018;28(2):e28011801.

https://doi.org/10.17061/phrp28011801

\section{Key points}

- People aged 60 years and older perceive yoga-based exercise to be an acceptable form of exercise

- Yoga-based exercise warrants investigation as a fall prevention strategy for older people

- Older people who preference yogabased exercise over the Otago Exercise Programme tend to be younger, less fearful of falling and view exercise more positively

\section{Abstract}

Objectives and importance of study: Yoga improves balance and mobility, and therefore has potential as a fall prevention strategy, yet its validity for preventing falls has not been established. The Otago Exercise Programme (OEP) and tai chi are proven to prevent falls. This study aimed to evaluate the perceptions and preferences of older people towards a yoga-based program with potential to decrease falls, to compare these perceptions to the views expressed about the OEP and tai chi, and to identify participant characteristics associated with a preference for the yoga program.

\section{Study type: Survey.}

Methods: Participants were 235 community-dwellers aged 60 years or older who were not participating or had not previously participated (within the past 10 years) in yoga-based exercise. Participants completed a self-report survey measuring demographics, physical activity level and attitude. They then viewed explanations of the yoga-based program, the OEP and tai chi. Participants completed the Attitudes to Falls-Related Interventions Scale (AFRIS) to measure program acceptability and identified their preferred program. Acceptability scores and preference were compared between the programs, and factors associated with yoga preference were identified with analysis of variance.

Results: The mean age of participants (69\% female) was 69.4 years (standard deviation 7.4). All programs were rated as equally acceptable $(p=0.17$ ), with AFRIS scores ranging from 28.1 to 29.4. Eighty-two people (35\%) preferred yoga, 32\% chose the OEP and 33\% chose tai chi. Overall, people who preferred yoga were significantly younger, healthier, less fearful of falling, and perceived exercise more positively than people who preferred the OEP ( $p$ values ranged from 0.03 to $<0.001$ ). The characteristics of people who preferred yoga and those who preferred tai chi did not vary significantly.

Conclusions: Yoga was perceived to be appropriate and was as popular as two validated fall prevention programs. Yoga warrants further investigation as a fall prevention strategy, particularly for 'younger' and healthier people aged 60 years or older. 


\section{Introduction}

The increased tendency to fall with age presents a major challenge to healthcare providers and health systems, as well as for older people and their carers. Falls affect a significant number of older people worldwide, and between $28 \%$ and $35 \%$ of people aged 65 years or older fall one or more times every year. ${ }^{1}$ Falls can result in disability, loss of mobility, reduced quality of life, fear of falling and even death. ${ }^{2}$

Programs that include exercises that challenge balance can reduce the risk of falls by almost $40 \% .^{3}$ Examples of the types of effective exercise programs for preventing falls include tai chi ${ }^{4}$ and the home-based Otago Exercise Programme (OEP). ${ }^{5}$ To date, uptake and adherence to these types of programs by older people has been low ${ }^{6}$, presenting a challenge to policy makers and clinicians caring for older people. A greater choice of fall prevention exercise options, and more targeted implementation strategies, may help address this shortfall in participation. ${ }^{7}$

A recent systematic review and meta-analysis conducted by the authors ${ }^{8}$ showed that participation in yoga-based exercise improves balance and physical mobility in people aged 60 years or older. These results indicate preliminary evidence of a fall prevention benefit from yoga participation. However, further research is needed to determine whether yoga-related improvements in balance and mobility can help prevent falls in older people.

Consideration of exercise preferences is crucial for guiding the implementation of effective programs for broad population health impact. Between 50\% and $90 \%$ of people reject falls-related interventions ${ }^{9}$; a better understanding of the reasons for this rejection may allow interventions to be offered in more acceptable ways to improve uptake and adherence. Previous research has shown that older people are more likely to start and continue to participate in exercise programs aimed at preventing falls if those programs emphasise overall positive impacts on health, mood and independence, rather than focusing only on preventing falls. ${ }^{9}$ Yoga is a holistic form of exercise that is likely to fit with the needs and preferences of many older people, but little research has been conducted to measure this.

This study aimed to evaluate the perceptions and preferences of older people towards a yoga-based program with potential to decrease falls, to compare these perceptions to the views expressed about two validated fall prevention exercise programs, and to identify participant characteristics associated with a preference for the yoga program. The specific study questions were:

1. Is a yoga-based exercise program acceptable to older community-dwelling people?

2. What are the characteristics of older people that are associated with a preference to participate in a yoga-based program with potential to decrease falls compared with the OEP and tai chi validated fall prevention exercise programs?

\section{Methods}

\section{Participants}

Community-dwelling people in Sydney, Australia, aged 60 years or older were invited to take part in the study between August 2013 and February 2014. Exclusion criteria were: current or previous (within the past 10 years) regular participation in a yoga-based exercise program, where regular was considered to be once a week for a period of 1 month within the past 10 years; residence in a hostel or nursing home; presence of a medical condition precluding exercise participation; cognitive impairment (Memory Impairment Screen score $\leq 5)^{10}$; or limited English language skills.

\section{Participant recruitment}

Participants were recruited through invitations to established networks of older people, advertisements in local newspapers, and online through a market research company. All participants were screened by phone or email, or assessed online, for eligibility before providing informed consent. The study design and procedures were approved by the Human Research Ethics Committee at the University of Sydney (protocol number 2013/430).

Participants completed a paper-based or online survey, which took approximately 30 minutes to complete. Paper surveys were mailed out with a self-addressed, reply-paid envelope to allow for ease of return to the researchers. Unreturned surveys were followed up with a reminder telephone call. The online survey was hosted on an external server and participants were emailed a web link to enable access.

\section{Study measures}

Demographic information collected included medical conditions, medications, fall history, need for assistance with activities of daily living, general health, and fear of falling as measured with the short Falls Efficacy Scale International (FES-I). ${ }^{11}$

Current physical activity participation was measured with the Incidental and Planned Exercise Questionnaire (IPEQ). ${ }^{12}$ This questionnaire measures participation in both structured and incidental physical activity. Perceptions and attitudes towards exercise were measured with the Exercise Benefits and Barriers Scale (EBBS). ${ }^{13}$ This 43 -item questionnaire consists of statements relating to benefits of, and barriers to, participating in exercise. Respondents rate their level of agreement with each statement on a four-point scale ranging from 'strongly agree' to 'strongly disagree'. Scores on the total questionnaire can range from 43 to 172 , with higher scores indicating a more positive perception of exercise.

After completing the demographic measures and background questionnaires, participants were presented with a series of pictures and explanations that illustrated 
the content and setting of the yoga-based fall prevention program (see Appendix 1, available from: hdl.handle. net/2123/17698). In response to this information, participants completed the Attitudes to Falls-Related Interventions Scale (AFRIS) ${ }^{14}$ to assess the acceptability of the proposed yoga program. AFRIS scores range from 6 to 42, with higher scores indicating higher program acceptability. Participants were also presented with a description and content pictures of two validated fall prevention exercise programs - the OEP 5 (which involves home-based strength and balance exercises) and a group-based tai chi program ${ }^{4}$ (see Appendix 1, available from: hdl.handle.net/2123/17698). The AFRIS was again completed for both interventions so that ratings of acceptability could be compared between the three different exercise programs. Lastly, participants were asked to nominate which program they would choose to participate in if they had to choose just one of the three.

\section{Data analysis}

Data were analysed using Stata statistical software (College Station, TX: StataCorp LP; Release 14).
Ratings of acceptability and preference were compared between the different programs. Differences in participant characteristics between those who preferred each of the three programs were identified with one-way analysis of variance (ANOVA) and Bonferroni post-hoc testing.

\section{Results}

Participants were 235 people (162 women) aged 60 to 96 years, with a mean age of 69 years (standard deviation [SD] 7.4). A total of 128 participants completed the survey online (54\%). Sixty-four participants (27\%) had experienced at least one fall in the past year, and 61 participants (26\%) self-rated a moderate fear of falling. Forty-seven participants (20\%) rated their balance as fair or poor, and 38 participants (16\%) rated their general health as fair or poor (Table 1). The survey respondents were representative of the broader state population of older people in terms of past falls, medication use, medical conditions and mobility limitations. ${ }^{6}$

Table 1. Characteristics of study participants $(N=235)$

\begin{tabular}{ll}
\hline Characteristic & Result \\
\hline Age, mean years (SD) & $69.4(7.4)$ \\
Female gender, $n(\%)$ & $162(69)$ \\
English spoken at home, $n(\%)$ & $227(97)$ \\
Lives alone, $n(\%)$ & $72(31)$ \\
Walking aid used, $n(\%)$ & $24(10)$ \\
Memory Impairment Screen, mean (SD) (max 8) & $7.4(0.9)$ \\
Fallen in the past 12 months, $n(\%)$ & $64(27)$ \\
Total number of medications, mean (SD) & $4.6(2.9)$ \\
Total number of medical conditions ${ }^{a}$, mean (SD) & $3.3(2.7)$ \\
Short FES-l' ${ }^{b}$ mean (SD) & $9.8(3.8)$ \\
IPEQ total physical activity, mean hours/week (SD) & $29.0(18.8)$ \\
Self-rated health fair/poor, $n(\%)$ & $38(16)$ \\
Self-rated balance fair/poor, $n(\%)$ & $47(20)$ \\
Exercise Benefits and Barriers Scale, mean totalc (SD) & $127.8(17.9)$ \\
\hline
\end{tabular}

FES-I = Falls Efficacy Scale - International; IPEQ = Incidental and Planned Exercise Questionnaire; SD = standard deviation

a Medical conditions selected from: arthritis (rheumatoid arthritis and osteoarthritis), osteoporosis, peripheral vascular disease, diabetes, asthma, upper gastrointestinal disease (ulcer, hernia, reflux), chronic obstructive pulmonary disease/emphysema, depression, angina/ ischaemic heart disease/heart attack, cognitive impairment/dementia, congestive heart disease, anxiety/panic disorder, hypertension, visual impairment, Parkinson's disease, hearing impairment, atrial fibrillation, cancer, stroke/transient ischaemic attack, gout

b Possible scores range from 7 to 28; higher scores indicate more concern about falling

c Possible scores range from 43 to 172; higher scores indicate more positive perception of exercise 
Yoga was rated as an acceptable program, with a mean AFRIS score of 29.1 out of 45 (SD 7.3). This was comparable to the ratings of acceptability for the other two programs, with AFRIS scores of 28.1 (SD 7.6) for the OEP and 29.4 (SD 7.7) for tai chi ( $p=0.17$ for comparisons).

Of the three exercise programs, 82 people (35\%) nominated yoga as their program preference, 75 (32\%) nominated the OEP and 78 (33\%) nominated tai chi. Participant characteristics stratified by program preference are listed in Table 2.
Several characteristics varied significantly between people who nominated the OEP as their preference and those who nominated either of the other two programs. Compared with OEP-preferers, people who preferred yoga were younger (mean age 68 years vs 72 years, $p=0.02$ ), had fewer medical conditions (3 vs $4, p=0.03$ ), had lower fear of falling (Short FES-I 9.4 vs 11.3, $p=0.005$ ) and viewed exercise more positively (EBBS 133 vs 120, $p<0.001$ ). No differences in participant characteristics were found between people who preferred yoga and those who preferred tai chi.

Table 2. Comparison of characteristics of people with different exercise program preferences

\begin{tabular}{|c|c|c|c|c|c|}
\hline Characteristics & $\begin{array}{l}\text { Preferred yoga } \\
(n=82)\end{array}$ & $\begin{array}{l}\text { Preferred OEP } \\
(n=75)\end{array}$ & $\begin{array}{l}\text { Preferred tai } \\
\text { chi }(n=78)\end{array}$ & $\begin{array}{l}\text { Between-group } \\
\text { comparison (ANOVA) }\end{array}$ & $\begin{array}{l}\text { Differences between } \\
\text { groups (post-hoc } \\
\text { Bonferroni) }\end{array}$ \\
\hline Age, mean years (SD) & $68.1(5.9)$ & $72.0(8.6)$ & $68.2(7.0)$ & $\begin{array}{l}F(2,232) 7.42 \\
p<0.001\end{array}$ & $\begin{array}{l}\text { Yoga/OEP, } p=0.02 \\
\text { OEP/tai chi, } p=0.03\end{array}$ \\
\hline Female gender, $n(\%)$ & $60(73)$ & $55(73)$ & $47(60)$ & $\begin{array}{l}F(2,232) 2.06 \\
p=0.13\end{array}$ & - \\
\hline $\begin{array}{l}\text { Memory Impairment Screen, } \\
\text { mean (SD) (max. 8) }\end{array}$ & $7.4(0.8)$ & $7.5(0.8)$ & $7.4(0.8)$ & $\begin{array}{l}F(2,232) 0.38 \\
p=0.68\end{array}$ & - \\
\hline $\begin{array}{l}\text { Fallen in the past } 12 \text { months, } \\
n(\%)\end{array}$ & $25(31)$ & $24(32)$ & $15(19)$ & $\begin{array}{l}F(2,232) 1.15 \\
p=0.32\end{array}$ & - \\
\hline $\begin{array}{l}\text { Total number of medical } \\
\text { conditions }^{a} \text {, mean (SD) }\end{array}$ & $3.0(2.9)$ & $4.1(2.9)$ & $2.8(2.1)$ & $\begin{array}{l}F(2,232) 5.17 \\
p=0.01\end{array}$ & $\begin{array}{l}\text { Yoga/OEP, } p=0.03 \\
\text { OEP/tai chi, } p=0.009\end{array}$ \\
\hline $\begin{array}{l}\text { Total number of medications, } \\
\text { mean (SD) }\end{array}$ & $4.3(2.8)$ & $5.3(3.3)$ & $4.1(2.7)$ & $\begin{array}{l}F(2,232) 3.54 \\
p=0.03\end{array}$ & OEP/tai chi, $p=0.03$ \\
\hline $\begin{array}{l}\text { IPEQ total physical activity, } \\
\text { mean hours/week (SD) }\end{array}$ & $30.0(19.9)$ & $26.1(18.8)$ & $30.8(17.2)$ & $\begin{array}{l}F(2,232) 1.37 \\
p=0.26\end{array}$ & - \\
\hline Short FES-I ${ }^{\mathrm{b}}$, mean (SD) & $9.4(3.2)$ & $11.3(4.8)$ & $8.6(2.5)$ & $\begin{array}{l}F(2,232) 10.99 \\
p<0.001\end{array}$ & $\begin{array}{l}\text { Yoga/OEP, } p=0.005 \\
\text { OEP/tai chi, } p<0.001\end{array}$ \\
\hline $\begin{array}{l}\text { Exercise Benefits and Barriers } \\
\text { Scale, mean total }{ }^{c}(S D)\end{array}$ & $132.6(17.5)$ & $119.6(17.8)$ & $130.6(15.9)$ & $\begin{array}{l}F(2,232) 13.04 \\
p<0.001\end{array}$ & $\begin{array}{l}\text { Yoga/OEP, } p<0.001 \\
\text { OEP/tai chi, } p<0.001\end{array}$ \\
\hline
\end{tabular}

- = not tested (ANOVA not significant); AFRIS = Attitudes to Falls-Related Interventions Scale; FES-I = Falls Efficacy Scale - International; IPEQ

$=$ Incidental and Planned Exercise Questionnaire; OEP = Otago Exercise Programme; SD = standard deviation

a Medical conditions selected from: arthritis (rheumatoid arthritis and osteoarthritis), osteoporosis, peripheral vascular disease, diabetes, asthma, upper gastrointestinal disease (ulcer, hernia, reflux), chronic obstructive pulmonary disease/emphysema, depression, angina/ ischaemic heart disease/heart attack, cognitive impairment/dementia, congestive heart disease, anxiety/panic disorder, hypertension, visual impairment, Parkinson's disease, hearing impairment, atrial fibrillation, cancer, stroke/transient ischaemic attack, gout

b Possible scores range from 7 to 28; higher scores indicate more concern about falling

c Possible scores range from 43 to 172; higher scores indicate more positive perception of exercise 


\section{Discussion}

These results show that a yoga-based exercise program designed to prevent falls was perceived to be equally as acceptable as two established and validated fall prevention programs. This result supports further investigation into yoga's role in the prevention of falls in older people.

Yoga appears to appeal to a certain type of older person, because there were significant differences in the characteristics of people who preferred yoga and those who preferred the OEP. Yoga-preferers were younger, healthier, had higher self-efficacy regarding falls and viewed exercise more positively. The characteristics of people who preferred the tai chi program did not vary significantly from those who preferred yoga.

The OEP is a home-based exercise program, in contrast to the group-based nature of both the yoga and tai chi programs. This may explain some of the difference in preferences.

This study has some limitations. Participants were recruited from established networks of older people and an online market research database, so may be more engaged and active than the population of older people more generally. For this reason, these results may not be applicable to frail older people in the community. Participants were required to make their decisions about the acceptability of the exercise programs after viewing a brief description and some photos of the programs, which may yield different perceptions than if the programs were viewed in real life, or if study participants tried the programs.

\section{Conclusion}

These results demonstrate the perceived acceptability of a yoga-based exercise program, which aims to improve balance and mobility, among community-dwelling older people aged 60 years or older. The results could assist with targeting different exercise programs to different people. Since yoga-based exercise was equally as acceptable as two established fall prevention programs, it should be investigated further for its role as a fall prevention strategy.

If shown to be effective in preventing falls in older people, yoga could be promoted alongside other exercise-based fall prevention strategies, and would increase the exercise choices available to older adults. These results may help to target yoga-based programs to older people in terms of the people most likely to take up and adhere to these programs.

\section{Acknowledgements}

This study was funded by the University of Sydney, Sydney Medical School New Staff/Early Career Researcher Scheme. The sponsor played no role in the study design, methods, subject recruitment, data collection, analysis or preparation of the article. AT and CS receive salary funding from National Health and Medical Research Council fellowship schemes.

\section{Peer review and provenance}

Externally peer reviewed, not commissioned

\section{Competing interests}

None declared

\section{Author contributions}

AT conceived the study, obtained funding and performed data analysis. SO and AT contributed to participant recruitment and data collection. All authors contributed to the methods, and contributed to and approved the written manuscript.

\section{References}

1. World Health Organization. World report on ageing and health. Geneva: WHO; 2015 [cited 2017 Dec 19]. Available from: apps.who.int/iris/ bitstream/10665/186463/1/9789240694811_eng. pdf?ua $=1$

2. Australian Institute of Health and Welfare. Australia's health 2016. Canberra: AlHW; 2016 [cited 2017 Dec 19]. Available from: www.aihw.gov.au/getmedia/9844cefb7745-4dd8-9ee2-f4d1c3d6a727/19787-AH16.pdf. aspx?inline $=$ true

3. Sherrington C, Michaleff ZA, Fairhall N, Paul SS, Tiedemann A, Whitney $\mathrm{J}$, et al. Exercise to prevent falls in older adults: an updated systematic review and metaanalysis. Br J Sports Med. 2017;51(24):1750-8.

4. Wolf SL, Barnhart HX, Kutner NG, McNeely E, Coogler $\mathrm{C}, \mathrm{Xu} \mathrm{T}$. Reducing frailty and falls in older persons: an investigation of tai chi and computerized balance training. J Am Geriatr Soc. 1996;44(5):489-97.

5. Robertson MC, Devlin N, Gardner MM, Campbell AJ. Effectiveness and economic evaluation of a nursedelivered home exercise program to prevent falls. 1: Randomised-control trial. BMJ. 2001;322:697-701. 
6. Centre for Health Advancement and Centre for Epidemiology and Research. New South Wales falls prevention baseline survey: 2009 report. Sydney: NSW Department of Health; 2010 [cited 2017 Dec 19]. Available from: www.health.nsw.gov.au/surveys/other/ Publications/falls-prevention-survey.pdf

7. Franco MR, Howard K, Sherrington C, Ferreira PH, Rose J, Gomes J L, Ferreira ML. Eliciting older people's preferences for exercise programs: a best-worst scaling choice experiment. J Physiother. 2015;61(1):34-41.

8. Youkhana S, Dean C, Wolf M, Sherrington C, Tiedemann A. Yoga-based exercise improves balance and mobility in people aged 60 and over: a systematic review and metaanalysis. Age Ageing. 2016;45(1):21-9.

9. Yardley L, Donovan-Hall M, Francis K, Todd C. Older people's views of advice about falls prevention: a qualitative study. Health Educ Res. 2006;21:508-17.
10. Lipton RB, Katz MJ, Kuslansky G, Sliwinski MJ, Stewart WF, Verghese J. Screening for dementia by telephone using the memory impairment screen. J Am Geriatr Soc. 2003;51:1382-90.

11. Yardley L, Beyer N, Hauer K, Kempen G, Piot-Ziegler C, Todd C. Development and initial validation of the Falls Efficacy Scale-International (FES-I). Age Ageing. 2005;34(6):614-19.

12. Delbaere K, Hauer K, Lord SR. Evaluation of the incidental and planned exercise questionnaire (IPEQ) for older people. Br J Sports Med. 2010;44(14):1029-34.

13. Sechrist K, Walker S, Pender N. Development and psychometric evaluation of the exercise benefits/barriers scale. Res Nurs Health. 1987; 10:357-65.

14. Yardley L, Donovan-Hall M, Francis K, Todd C. Attitudes and beliefs that predict older people's intention to undertake strength and balance training. J Gerontol B Psychol Sci Soc Sci. 2007;62B(2):119-25.

\section{Copyright: (c) (i) (2)}

(C) 2018 Tiedemann et al. This article is licensed under the Creative Commons Attribution-NonCommercial-ShareAlike 4.0 International Licence, which allows others to redistribute, adapt and share this work non-commercially provided they attribute the work and any adapted version of it is distributed under the same Creative Commons licence terms. See: www.creativecommons.org/licenses/by-nc-sa/4.0/ 